\title{
Criteria for Selecting GLONASS Satellites \\ to Reduce the Systematic Error in Determining the Coordinates in the Horizon Plane
}

Vladimir B. Pudlovsky* Russian Metrological Institute of Technical Physics and Radio Engineering Mendeleevo, Moscow region, 141570, Russia

Received 23.06.2018, received in revised form 02.07.2018, accepted 13.07.2018

The analysis of the effect of systematic errors in pseudorange measurements on the accuracy of determination the coordinates in the plane of the horizon is presented. The criteria for selecting navigation satellites GLONASS are proposed to minimize the systematic components of the error of the planned coordinates, determined on the basis of the method of least squares. The results of checking the effect of selection of satellites when processing real pseudorange measurements by signals of the GLONASS system are presented.

Keywords: navigation receiver, systematic error, coordinates in the horizon plane.

Citation: Pudlovsky V.B. Criteria for selecting GLONASS satellites to reduce the systematic error in determining the coordinates in the horizon plane, J. Sib. Fed. Univ. Eng. technol., 2019, 12(1), 72-80. DOI: 10.17516/1999-494X-0077.

\section{Критерии выбора спутников ГЛОНАСС \\ для снижения систематической погрешности определения плановых координат}

В.Б. Пудловский Всероссийский научно-исследовательский институт физико-технических и радиотехнических измерений Россия, 141570, Московская область, Менделеево

Представлен анализ влияния систематических погрешностей измерений псевдодальностей на точность определения плановых координат (в плоскости горизонта потребителя). Предложены критерии выбора навигационных космических аппаратов ГЛОНАСС для

(C) Siberian Federal University. All rights reserved

This work is licensed under a Creative Commons Attribution-NonCommercial 4.0 International License (CC BY-NC 4.0).

* Corresponding author E-mail address: pudlovskiy@vniiftri.ru 
минимизации систематических составляющих погрешности плановых координат, определяемых на основе метода наименьших квадратов. Приведень результаты проверки эффекта отбора спутников при обработке реальных измерений псевдодальностей по сигналам системы ГЛОНАСС.

Ключевые слова: навигациионая аппаратура, систематическая погрешность, плановые координатьл.

\section{Introduction}

The accuracy of coordinates and height estimating of navigation receiver only by global navigation satellite system (GNSS) signals without any additional information i.e. in the absolute mode is one of the most important metrological characteristic.

The accuracy of the coordinates is generally estimated in an assumption that pseudorange biases to navigational space vehicles (NSV) are equally distributed according to zero-mean normal law and are not correlated between each other [1, 2]. Dilution of Precision (DOP) is generally used as a main criterion of prior coordinates error estimation for these conditions. However, such pseudorange bias idealization is not always justified.

Even for measurements by NSV signals of one GNSS, mainly of GLONASS, the most significant budget components of equivalent range error are distributed in a way that differs from normal and non-zero mean value. It is confirment by effectiveness of various differential and relative navigation methods $[1,2]$.

For higher precision of navigation receiver (NR) in an absolute mode one should use methods decreasing the influence of systematic pseudorange biases without any additional information.

\section{Problem definition}

In several algorithms of pseudorange processing of NR mainly used by Russian engineers the meaning of DOP is not used at all for NSV selecting ("all in view" mode). Moreover, one often applies algorithms based on the least square method (LSM) for measurements processing on all visible NSV to define the receiver coordinates.

It is well known that the aim of navigation task (NT) solution in the receiver is the receivers condition vector definition $\mathbf{x}$ (coordinates and height of the receiver antenna as well as the estimation of the receiver timescale shift relatively to the system time scale) according to the pseudorange measurements $R_{j}$ not less than to $4 \mathrm{NS}(j=1 \ldots n, n \geq 4)$. Vector form of these measurements after linearization about true value $\mathbf{x}$ is represented in the following way $[1,2]$

$$
\mathbf{y}=\mathbf{G x}+\boldsymbol{\varepsilon},
$$

where $y_{j}=R_{j}-\rho_{j}-d_{j}-$ pseudorange measurement result to $j$-NS considering the correction by the value of prior range $\rho_{j}$ to the NS and the sum of different modeling corrections $d_{j}$; $\mathbf{G}=\left[\begin{array}{cccc}a_{1} & b_{1} & g_{1} & 1 \\ a_{2} & b_{2} & g_{2} & 1 \\ \vdots & \vdots & \vdots & \vdots \\ a_{n} & b_{n} & g_{n} & 1\end{array}\right]=[\mathbf{a} \mathbf{b} \mathbf{g} \mathbf{1}]-$ the matrix of partial pseudorange derivatives on condition 
vector; $\boldsymbol{\varepsilon}$ - vector of measurement errors the mean value of which is generally assumed as $\mathbf{r}=\mathrm{E}[\boldsymbol{\varepsilon}]$ and variance matrix as $\mathbf{C}=\mathrm{E}\left[\boldsymbol{\varepsilon}^{\mathrm{T}} \boldsymbol{\varepsilon}\right]$.

The typical algorithm of navigation task solution using LSM for the number of measurements more than 4 is described like this [2]:

$$
\hat{\mathbf{x}}=\left(\mathbf{G}^{\mathrm{T}} \mathbf{G}\right)^{-1} \mathbf{G}^{\mathrm{T}} \mathbf{y}
$$

where $\hat{\mathbf{x}}$ - the estimation of condition vector (CV) according LSM algorithm.

Equations (1) and (2) allow specifying the error of $\mathrm{CV}$ estimation in the following way

$$
\hat{\mathbf{x}}-\mathbf{x}=\left(\mathbf{G}^{\mathrm{T}} \mathbf{G}\right)^{-1} \mathbf{G}^{\mathrm{T}} \boldsymbol{\varepsilon}
$$

The hypothesis is often assumed on the absence of systematic errors in measurements (1), supposing the mean value $\varepsilon$ as zero

$$
\mathbf{r}=\mathrm{E}[\boldsymbol{\varepsilon}]=0
$$

Taking into consideration the assumption, $\mathrm{CV}$ estimation will be unbiased as well that can be traced from (3)

$$
\boldsymbol{\Delta}=\mathrm{E}[\hat{\mathbf{x}}-\mathbf{x}]=\left(\mathbf{G}^{\mathrm{T}} \mathbf{G}\right)^{-1} \mathbf{G}^{\mathrm{T}} \mathbf{r}=0
$$

where $\boldsymbol{\Delta}=\left[\Delta_{1} \Delta_{2} \Delta_{3} \Delta_{4}\right]^{\mathrm{T}}-$ vector of mean values of $\mathrm{CV}$ estimation errors.

In topocentric (local) coordinates system its components have the following meaning:

$\Delta_{1}=\mathrm{E}\left[\hat{x}_{1}-x_{1}\right]-$ mean value of longitude error,

$\Delta_{2}=\mathrm{E}\left[\hat{x}_{2}-x_{2}\right]-$ mean value of latitude error,

$\Delta_{3}=\mathrm{E}\left[\hat{x}_{3}-x_{3}\right]-$ mean value of height error,

$\Delta_{4}=\mathrm{E}\left[\hat{x}_{4}-x_{4}\right]-$ mean value of estimation error of NR timescales shift in relation to GNSS timescale.

Note also that, through the diagonal elements of $q_{i i}$ matrices $\left(\mathbf{G}^{\mathrm{T}} \mathbf{G}\right)^{-1}$ often determine the values of the DOP, for example in the following form [2]:

$$
\begin{aligned}
& \text { GDOP }=\left(\operatorname{tr}\left[\left(\mathbf{G}^{\mathrm{T}} \mathbf{G}\right)^{-1}\right]\right)^{0,5}=\left(q_{11}+q_{22}+q_{33}+q_{44}\right)^{0,5}, \\
& P D O P=\left(q_{11}+q_{22}+q_{33}\right)^{0,5}, \\
& H D O P=\left(q_{11}+q_{22}\right)^{0,5},
\end{aligned}
$$

where $G D O P$ - geometric dilution of precision NT solution; $P D O P$ - position dilution of precision in three-dimensional space; $H D O P$ - horizontal dilution of precision in the horizon plane.

The analysis of the pseudorange bias budget shows that condition $\mathbf{r}=0$ is rarely met in practice especially for short periods of coordinates definition with the help of NR.

It follows that the fulfillment of (4) for $\mathbf{r} \neq 0$ is possible only by determining the elements of the matrix G. Hence the purpose of the paper can be formulated as follows: to solve the NT, it is necessary to determine such a configuration of the NSV that will ensure a minimum 
displacement of the estimated CV components, if there is some information on the components $\mathbf{r} \neq 0$.

\section{Theory}

If solution (2) exists then

$$
\left(\mathbf{G}^{T} \mathbf{G}\right)^{-1} \neq 0 \text {. }
$$

Thus, condition (4) for $\mathbf{r} \neq 0$ can be formally fulfilled if

$$
\mathbf{G}^{\mathrm{T}} \mathbf{r}=[\mathbf{a} \mathbf{b} \mathbf{g} \mathbf{1}]^{\mathrm{T}} \mathbf{r}=0 .
$$

It is evident that for $\mathbf{r}$ different from zero its projection on axes of 4-dimentional space of coordinates to be defined cannot be simultaneously zero.

Due to this reason it is better to be limited further by conditions finding under which the compensation of systematic pseudorange bias is possible only for the coordinates in plane i.e. the following must be fulfilled

$$
\Delta_{1}=\Delta_{2}=0
$$

To put (4) as a normal system

$$
\begin{aligned}
& \mathbf{G}^{T} \mathbf{G} \boldsymbol{\Delta}=\mathbf{G}^{T} \mathbf{r}, \\
& \mathbf{G} \boldsymbol{\Delta}=[\mathbf{a} b \mathbf{g} \mathbf{1}] \mathbf{\Delta}=\mathbf{r} .
\end{aligned}
$$

Condition (6) results in orthogonality of vector $\mathbf{r}$ to two first basis vectors of its resolution that is expressed through scalar product

$$
(\mathbf{a}, \mathbf{r})=(\mathbf{b}, \mathbf{r})=0 \text {. }
$$

Then one should understand when conditions (8) are fulfilled for such type of $\mathbf{r}$ all the components of which differs from 0 .

First variant is when $\mathbf{r}$ components are close regarding absolute value. Then columns $\mathbf{1}$ and $\mathbf{r}$ of system (7) are linearly dependent. Therefore the solution of (7), for instance, according Kramer's rule will result in $\Delta_{1}=\Delta_{2}=\Delta_{3}=0$, i.e. there won't be any shift on all the coordinates and height. Systematic error $\Delta_{4}$ of NR timescale shift regarding GNSS scale will be the only to increase.

Considering the nature of the most considerable errors composing pseudorange estimation budget two more variants of $\mathbf{r}$ component correlation are to be described:

A) components are positive and similar for NSV pairs;

B) components are positive and proportional to NSV elevation angels $\gamma_{j}$.

Thus condition (8) is to be presented in the following way:

for variant $\mathrm{A}$ :

$$
\begin{aligned}
& r_{1}\left(a_{1}+a_{2}\right)+\ldots+r_{n / 2}\left(a_{n-1}+a_{n}\right)=0, \\
& r_{1}\left(b_{1}+b_{2}\right)+\ldots+r_{n / 2}\left(b_{n-1}+b_{n}\right)=0
\end{aligned}
$$


for variant B:

$$
\sum_{j=1}^{n} r_{j}\left(\gamma_{j}\right) \cdot a_{j}=\sum_{j=1}^{n} r_{j}\left(\gamma_{j}\right) \cdot b_{j}=0
$$

The expression for partial derivatives $a_{j}, b_{j}$ in topocentric (local) coordinates system and in the polar coordinates system [2] is defined in the following way:

$$
\begin{aligned}
& a_{j}=\sin \alpha_{j} \cdot \cos \gamma_{j}, \\
& b_{j}=\cos \alpha_{j} \cdot \cos \gamma_{j},
\end{aligned}
$$

where $\alpha_{j}$ - azimuth angel of NSV sight line.

Considering this way of specifying $a_{j}$ and $b_{j}$ one can easily notice that it is enough to form NSV pairs according to central symmetry in the plane (the difference by azimuth on $180^{\circ}$ and equal elevation angels for NSV pair) to fulfill (9) (variant A).

Functional dependence $r_{j}\left(\gamma_{j}\right)$ (variant B) is specific for pseudorange bias components description at the expense of refraction in ionosphere and troposphere. For instance, to recalculate the value of zenith delay in troposphere $Z T D_{j}$ for slant path $Z_{j}$ it is generally used so-called "mapping function" $m\left(\gamma_{j}\right)[3]$.

$$
Z_{j}\left(\gamma_{j}\right) \cong m\left(\gamma_{j}\right) Z T D_{j}
$$

In the same way one should consider the influence of the slant path while calculating the delay in ionosphere in the well-known Klobuchar's algorithm [2]. In the first approximation function $m\left(\gamma_{j}\right)$ will be

$$
m\left(\gamma_{j}\right)=1 / \sin \left(\gamma_{j}\right)
$$

The conclusion is that all systematic errors of zenith delays calculation in ionosphere and troposphere will only increase for slant paths of NSV signal propagation in the proportion close to $\sin ^{-1}\left(\gamma_{j}\right)$. However, these systematic bias can be considered close value for NSV with the equal elevation angles $\gamma_{j}$. Thus, conditions (9) and (10) are possible for the same NSV pairs selected according to central symmetry.

The analysis of condition (8) regarding (11) explains that these conditions are met not only for the given NSV pairs. It is evident that condition (8) is fulfilled by any of NSV for which $\gamma_{j} \approx 90^{\circ}$. Consequently, without influence on coordinate's error one can include several additional NSV observed in zenith relative to NR in working constellation if it is provided by orbital NSV group from one or several GNSS.

\section{Experiment results}

The approbation of the influence of the compensation of equal systematic pseudorange bias in GLONASS NSV pairs was conducted on base of the coordinates calculation in the plane comparison with the use of software package RTKLIB v.2.4.2 [4], where the algorithm of definition of absolute coordinates and height with the least square method was partially implemented.

As reference information there were used the measurement data by GLONASS ranging code (open civil code) only in L1 frequency band. The measurements with period $1 \mathrm{~s}$ are obtained in RINEX 
format version 3.03 from NR PolaRx5 (Septentrio), with the help of antenna positioned on the territory of FSUE "VNIIFTRI" in the point with given coordinates.

For further processing the data was used that was recorded during 24 hours dt. 15.03.2018.

NSV selection parameters were formed regarding the following acceptable deviation from satellites search conditions: not more than $10^{\circ}$ on elevation angle; not more than $30^{\circ}$ on azimuth.

For the location of the NR antenna for the day 15.03.2018, the selection conditions for two pairs of NSV at the viewing angles were made for several time intervals (lasting more than 5 minutes each) with a total duration of more than $400 \mathrm{~min}$. In this selection, an additional condition was used: the difference in the azimuth angle of the viewing vector for NSV from different pairs should exceed $40^{\circ}$.

Moreover one can notice 2 and sometimes 3 pairs of satellites centrally-symmetric regarding NR as well as generally one satellite close to zenith $\left(\gamma>75^{\circ}\right)$. The total sum of GLONASS NR satellites amounts from 8 to 10 .

To assess the probability of fulfilling the selection condition for "centrally symmetric" NSVs at other geographic locations, the above-mentioned conditions for the mutual sighting of the GLONASS satellites were simulated for an interval of 8 days. The results of calculating the probability of selection of at least two pairs of NSV are presented in Table 1.

The efficiency of only centrally-symmetric NSV choice for plane coordinates definition was compared to errors received during processing of all visible GLONASS satellites with elevation angles $\gamma>5^{\circ}$. The constellations from 4 NSV (2 pairs) has been selected as well as 5 NSV ( 2 pairs and one NSV close to zenith).

Fig. 1 shows the location example of 10 GLONASS satellites in relation to NR at 00:00 (UTC) for selecting two pairs ( $R 02, R 08, R 16, R 23$ numbers) and zenith NSV ( $R 01)$.

Some results of calculations of the error in determining the coordinates in the plan using the RTKLIB program package using the LSM algorithm are presented in Fig. 2 and in Table 2.

Fig. 2 represents the results of coordinates definition with the LSM during the period of 00:0000:05 (UTC). Blue dots mark the coordinates received according to 9 NSV signals considering measurements correction by a model. Red dots mark the coordinates received according to 5 selected

Table 1. Probability of observation of two pairs of satellites GLONASS

\begin{tabular}{|c|c|c|}
\hline $\begin{array}{c}\text { Coordinates of the point, degrees } \\
\text { latitude, longitude }\end{array}$ & $\begin{array}{c}\text { The total observation interval of } \\
\text { two pairs, min }\end{array}$ & $\begin{array}{c}\text { Probability of observation of at } \\
\text { least two pairs in 8 days }\end{array}$ \\
\hline $\begin{array}{c}56,02 \mathrm{~N} \\
37,22 \mathrm{E}\end{array}$ & 2919 & 0,25 \\
\hline $\begin{array}{c}59,93 \mathrm{~N} \\
30,30 \mathrm{E}\end{array}$ & 4597 & 0,40 \\
\hline $\begin{array}{c}68,97 \mathrm{~N} \\
33,09 \mathrm{E}\end{array}$ & 4851 & 0,42 \\
\hline $\begin{array}{c}44,50 \mathrm{~N} \\
34,16 \mathrm{E}\end{array}$ & 3333 & 0,29 \\
\hline $\begin{array}{c}56,02 \mathrm{~N} \\
92,87 \mathrm{E}\end{array}$ & 2802 & 0,24 \\
\hline $\begin{array}{c}80,0 \mathrm{~N} \\
100,0 \mathrm{E}\end{array}$ & 3053 & 0,26 \\
\hline
\end{tabular}




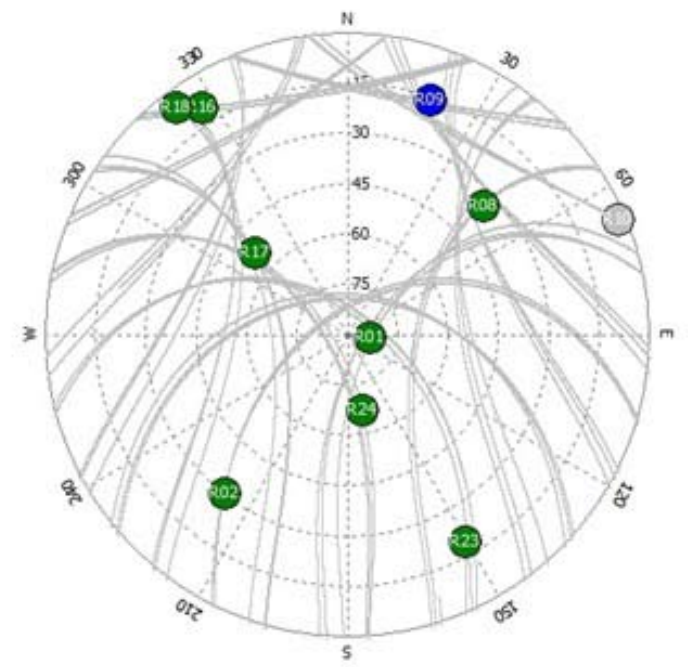

Fig. 1. The view of GLONASS satellites at 00:00 (UTS)

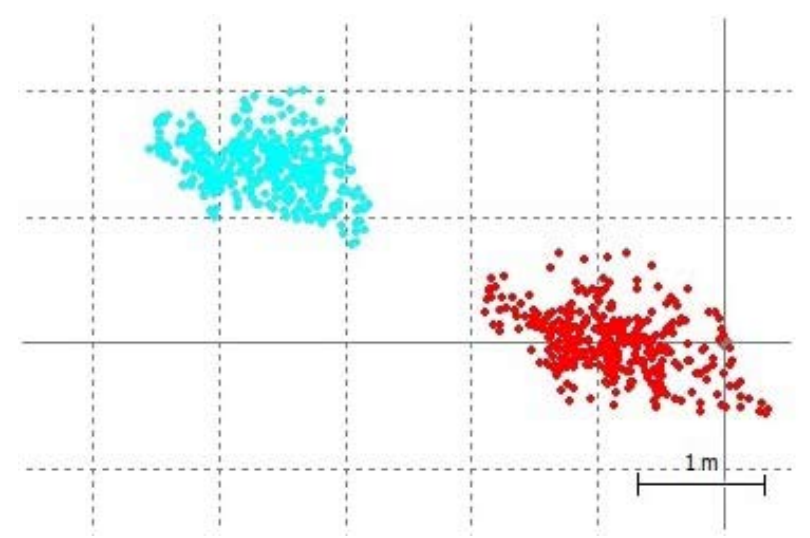

Fig. 2. Coordinates in the horizon plane according to pseudorange measurements by 5 (red dots) and 9 (blue dots) of GLONASS satellites

NSV (two pairs and one NSV in zenith) without using measurements correction by a model. The point coordinates accepted as standard are shown as intersection of grid full lines.

In the first column of Table 2 the number of NSV 4, 5 or 6 means applying the procedure of satellites pair selection by central symmetry criterion plus one in zenith (for 5 selected NSV).

There were used all pseudorange measurements available at the given time to define the coordinates for NSV number 9 or 10.

Symbols $\sigma_{1}$ and $\sigma_{2}$ stand for standard deviation relating the mean values $\Delta_{1}$ and $\Delta_{2}$ of error of coordinates under definition.

The capabilities of the RTKLIB package allow you to enable or disable models for correcting pseudoranges due to the influence of the troposphere and the ionosphere when processing measurements conducted in the one frequency band, which is also reflected in Table 2 in the column "Correction by models". 
Table 2. Coordinate errors

\begin{tabular}{|c|c|c|c|c|c|c|c|c|}
\hline \multirow{2}{*}{$\begin{array}{l}\text { The } \\
\text { number } \\
\text { of NSV }\end{array}$} & \multirow{2}{*}{$\begin{array}{c}\text { Correction } \\
\text { by } \\
\text { models }\end{array}$} & \multicolumn{2}{|c|}{ DOP } & \multicolumn{2}{|c|}{$\begin{array}{c}\text { Longitude } \\
\text { error, m }\end{array}$} & \multicolumn{2}{|c|}{$\begin{array}{l}\text { Latitude } \\
\text { error, } \mathrm{m}\end{array}$} & \multirow{2}{*}{$\begin{array}{c}\text { Time } \\
\text { (UTC), } \\
\text { h:min }\end{array}$} \\
\hline & & PDOP & HDOP & $\Delta_{1}$ & $\sigma_{1}$ & $\Delta_{2}$ & $\sigma_{2}$ & \\
\hline 9 & On & 1,5 & 1,0 & $-3,65$ & 0,42 & 1,41 & 0,23 & $00: 00$ \\
\hline 9 & Off & 1,5 & 1,0 & 0,16 & 0,20 & $-0,41$ & 0,27 & 00:00 \\
\hline 4 & Off & 3,5 & 1,2 & $-1,84$ & 0,64 & 0,78 & 0,27 & 00:00 \\
\hline 5 & Off & 2,0 & 1,2 & $-0,90$ & 0,46 & 0,01 & 0,25 & 00:00 \\
\hline 10 & On & 1,4 & 0,8 & $-2,68$ & 0,40 & 1,24 & 0,30 & $00: 05$ \\
\hline 10 & Off & 1,4 & 0,8 & $-2,48$ & 1,62 & $-0,27$ & 0,32 & 00:05 \\
\hline 4 & Off & 3,4 & 1,2 & $-1,51$ & 0,49 & 1,41 & 0,52 & 00:05 \\
\hline 5 & Off & 2,0 & 1,2 & $-1,19$ & 0,66 & 0,66 & 0,55 & 00:05 \\
\hline 9 & On & 1,8 & 0,8 & 2,61 & 0,23 & $-1,34$ & 0,45 & 03:40 \\
\hline 9 & Off & 1,8 & 0,8 & 2,64 & 0,23 & $-2,42$ & 0,44 & 03:40 \\
\hline 6 & On & 4,9 & 1,0 & 1,81 & 0,28 & $-1,71$ & 0,44 & $03: 40$ \\
\hline 6 & Off & 4,9 & 1,0 & 1,65 & 0,29 & $-2,27$ & 0,47 & 03:40 \\
\hline
\end{tabular}

The values of the DOP are calculated only for the working constellation of NSV, whose pseudorange measurements are used in determining the coordinates.

The analysis of received errors of height definition has not been performed in the course of the work.

The results of coordinates definition error in Table 2 are shown as an example and do not cover the whole scope of processed data received within the period of 24 hours.

\section{Discussion of results}

For the GLONASS orbital constellation of 24 satellites, the probability of observing two pairs of "centrally symmetric" NSV is from 0.24 to 0.4 or about 400 minutes per day.

The analysis of coordinates definition errors described in Table 2 as well as those received after other measurements have been processed verifies the advantage of NSV selection for partial compensation of defining coordinates shift from the real position of NR antenna.

However it is impossible to fully eliminate the shift of coordinates by NSV selecting. One of the reasons is likely to be the significant difference of pseudorange biases for the pair of selected NSV GLONASS.

As one can see from Table 2 reducing the NSV number the measurement of which were used for coordinate definition can possibly increase the coordinate error dispersion. It is particularly notable during comparison of the values of standard deviation longitude error for operating constellations from 4 and 9 NSV within the period of 00:00 - 00:05. Moreover the mean coordinate value received for selected NSV is as a rule closer to the primary standard.

On the other hand elimination of relatively small NSV number from visible NSV improves the coordinate definition accuracy only to a small extent that might be seen from the errors comparison for operating constellations from 6 and 9 NSV within the period of 03:40-03:45 (Table 2). It is easy to understand that in this case the main part of the satellites in an operating constellation has not 
changed while pseudorange bias for eliminated and selected NSV is likely to be comparable by the value.

A comparative analysis of the DOP values for a small amount of NSV in the working constellation (about half of the observed satellites) shows that the value of PDOP can not serve as a reliable criterion for the accuracy of the determination of coordinates (in the plane of the horizon). The value of HDOP gives a more adequate estimate of the coordinate error in the plane of the horizon. However, this DOP, calculated in accordance with (5), does not allow to take into account the influence of systematic errors in pseudorange measurements.

Based on the results of analysis of the processed data, it should be separately noted that the compensation of the displacement of the determined coordinates in the in the horizon plane due to selection of the NSV appears more noticeably in the absence (shutdown) of correction of pseudorange measurements by the models included in the RTKLIB software package.

\section{Conclusions}

Thus the research describes the possibility of pseudorange bias compensation in the pair of NSV during coordinates definition in horizontal plane by the least squares method algorithm. The maximum effect of such compensation can be achieved in the following conditions:

coincidence of absolute value of pseudorange bias in each NSV part;

central-symmetric position of two or more NSV pairs in the projection of spherical coordinates system to horizontal plane (the difference according azimuth to $180^{\circ}$ and equal elevation angels for NSV pair);

The usage of NSV located in zenith does not influence the shift of defining coordinates in the plane and allows applying NSV pair with the same elevation angles in different pairs.

The influence of compensation effect of systematic bias is notable during the processing of real pseudorange measurements for selected NSV GLONASS. However, it is impossible to eliminate the shift of defining coordinates.

The use of the DOP values as a criterion for choosing the composition of the working constellation of the NSV does not take into account the influence of systematic errors in pseudorange measurements and does not allow to adequately estimate the accuracy of the coordinates in the horizon plane.

The relevant objective for coordinates definition in absolute mode of NR is to develop new algorithms considering pseudorange bias shift and correlation.

\section{References}

[1] GLONASS: Design and Operating Principles, Perov A.I. and Kharisov V.N., Moscow: Radiotekhnika, 4th ed., revised, 2010. 800 p. (In Russian)

[2] Sanz Subirana J., Juan Zornoza J.M., Hernández-Pajares M., GNSS Data Processing Book, Vol. I: Fundamentals and Algorithms, TM-23/1. Noordwijk: ESA Communications, 2013. 223 p.

[3] Pudlovskiy V.B. Tropospheric delay: models and estimation error. The series "Radio communication and radio navigation". Release 3. Radio navigation technology. Perov A.I., Vlasov I.B. Moscow: Radiotekhnika, 2013. p. 50-54. (In Russian)

[4] RTKLIB [Electronic resource] RTKLIB: An Open Source Program Package for GNSS Positioning. - Acess: http://www.rtklib.com/ (date of the application: 14.08.2017). 